\title{
Seroprevalence and Risk Factors of Syphilis Infection among Antiretroviral Therapy Naive HIV Patients at the Cape Coast Teaching Hospital, Ghana
}

\author{
Prince Asare-Bediako1, Kwabena Dankwa², Daniel E. Azumah3 ${ }^{3}$, Samuel V. Nuvor ${ }^{2 *}$ \\ ${ }^{1}$ Department of Biomedical Sciences, School of Biological Sciences, University of Cape Coast, Cape Coast, Ghana \\ ${ }^{2}$ Department of Microbiology and Immunology, School of Medical Sciences, University of Cape Coast, Cape Coast, Ghana \\ ${ }^{3}$ Cape Coast Teaching Hospital, Cape Coast, Ghana \\ Email: *s.v.nuvor@uccsms.edu.gh
}

How to cite this paper: Asare-Bediako, P., Dankwa, K., Azumah, D.E. and Nuvor, S.V. (2018) Seroprevalence and Risk Factors of Syphilis Infection among Antiretroviral Therapy Naive HIV Patients at the Cape Coast Teaching Hospital, Ghana. World Journal of AIDS, 8, 76-89.

https://doi.org/10.4236/wja.2018.83007

Received: July 13, 2018

Accepted: September 8, 2018

Published: September 11, 2018

Copyright (c) 2018 by authors and Scientific Research Publishing Inc. This work is licensed under the Creative Commons Attribution International License (CC BY 4.0).

http://creativecommons.org/licenses/by/4.0/

\begin{abstract}
Diagnosis of sexually transmitted infections is very important considering the spread of HIV and the extensive use of highly active antiretroviral therapy worldwide. This will assist in planning of treatment schedule in controlling these infections. The study therefore aimed at determining the prevalence of syphilis in HIV positive antiretroviral therapy naive patients in Cape Coast and the associated risk factors involved in infection. A cross-sectional study was carried out using initial HIV rapid and confirmation tests, and then Venereal Disease Research Laboratory test with the Ultra Rapid Test Kits for syphilis. Demographic data, risky sexual behaviours capable of co-transmission of both HIV and Syphilis, were also collected through the use of questionnaires. In all, $150 \mathrm{HIV}$ positive antiretroviral naive subjects were studied and $15(10 \%)$ were positive for VDRL test, with females (6.00\%) and males (4.00\%), who were mainly within the age group of $20-39$ years. A significant number of males $(p=0.019)$ and females $(p=0.015)$ participants were not smoking with a fewer number of the females $(p=0.002)$ having multiple sexual partners. Also a smaller number of those who were infected with the bacteria $(\mathrm{p}=0.004)$ did not support the control of sexually transmitted infection $(p=0.022)$. The result showed that co-infection of Syphilis in HIV positive antiretroviral therapy naive patients persists in the Cape Coast Metropolis, which is an indication of prominence of STIs that require further study on a larger scale to ascertain the extent of co-infection and to formulate policy for treatment to help minimize the rate of infection.
\end{abstract}




\section{Keywords}

Seroprevalence, HIV, Syphilis, Risk Factors, Antiretroviral Therapy

\section{Introduction}

Sexually Transmitted Infections (STIs) are of a major public health importance especially in the developing countries. Although several drugs are available for the treatment of the infections, it has been difficult to control as a result of the emergence of Human Immunodeficiency Virus (HIV) infection [1]. HIV infection severely weakens the immune system of infected individuals who are therefore exposed to opportunistic infections including sexually transmitted infections. The co-infection of sexually transmitted infections and HIV may be having a profound detrimental effect especially among the youth in developing countries. Syphilis and HIV appear to be the most reported STIs, although there has been a worldwide decrease in the prevalence of syphilis by the year 2000 [2]. The resurgence of cases of syphilis has been observed in the last decade [3] and about 357 million people aged between 15 and 49 are infected every year with Chlamydia, Gonorrhea, Syphilis, or Trichomoniasis with more than $95 \%$ of antenatal care attendees tested for syphilis [4]. It has been observed in previous years, that the rate of reported syphilis cases among men ( 15.6 cases per 100,000 males) was much higher than the rate among women (1.9 cases per 100,000 females), and men accounted for a large majority (88.9\%) of syphilis cases [5]. Also 76.1 million people have become infected with HIV since the start of the epidemic [6], and about 36.7 million people globally were living with HIV with 35.0 million people dying from AIDS-related illnesses since the start of the epidemic [7]. The development of both HIV and Syphilis infection in patients is complex and remains not well elucidated in spite of about 2 decades of clinical experience with co-infected patients. It has been established that primary syphilis facilitates both the transmission and acquisition of HIV infection [8] [9]. However, the relationship between the two infections among individuals is a concern.

In Ghana, data on population about HIV and Syphilis are very scanty. A few studies carried out on syphilis and HIV in some part of the country such as among truck drivers at Tema metropolis showed the prevalence of $3.8 \%$ and $0.98 \%$ respectively [10]. The prevalence of HIV and Syphilis infections and the associated factors among blood donors in Koforidua also showed $4.5 \%$ and $15.3 \%$ respectively [11]. There were also the seroprevalence of syphilis (8.2\%) in blood donors at Komfo Anokye Teaching Hospital [12]. Again the prevalence of syphilis and HIV among prison inmates and officers at Nsawam and Accra was $16.5 \%$ and $5.9 \%$ respectively [13]. Nevertheless, recent data by the National AIDS Control Programme, a body responsible for carrying out sentinel survey of communicable diseases in the country showed the national syphilis prevalence rate of the country to be $0.2 \%$ [14]. This is an indication that syphilis infection is 
nationwide and may be high in urban areas since these areas are highly populated with economic and social activities.

Recently, Ghana has shown the Central Region as the leading region in syphilis cases in the country. And the sentinel report presented by Ghana AIDS Commission in March 2012 showed that Cape Coast had 9.6\% of HIV prevalence, being the highest in the country [14]. The high rates of infection of these two diseases suggest a notable evidence of co-infection among individuals, which is definitely affecting the economy in terms of reducing productivity. There is also reduction in the normal health states of population rendering it dangerous to live in some parts of the country. The main objective was to determine the prevalence of syphilis and the associated factors in HIV positive but antiretroviral therapy naïve patients in Cape Coast metropolis.

\section{Methods}

\subsection{The Study Area}

The study was carried out at the Cape Coast Teaching Hospital where majority of people visit for Medical care. The teaching hospital is a 400 bed facility in the Central Region of Ghana and the main referral hospital that serves the Region as well as parts of Western and Ashanti Regions. It also provides facilities for the training of medical personnel such as doctors and nurses from the University of Cape Coast. It has HIV clinic which has been providing services for patients since 2006.

Cape Coast is the capital town of the Central Region which has a settlement population of about 169,894 [15]. Vegetation of the study area (Cape Coast) is mainly secondary forest with thickets and shrubs growing to an average height of $4.5 \mathrm{~m}$. There is also a coastline which is about $13 \mathrm{~km}$ long. Cape Coast is a humid area with mean monthly relative humidity varying between $85 \%$ and $99 \%$. The sea breeze has a moderating effect on the local climate. The metropolis is bounded on the east by Abura-Asebu-Kwamankese District, West by Komenda-Edina-Eguafo-Abrem District, and south by the Gulf of Guinea and north by Twifo Heman Lower Denkyira District. The city is one of the communities within the metropolis along the southern coast, and as such, fishing is one of its major economic activities. The Cape Coast Metropolis is the central focus of tourism in the region. Within the Metropolis are many sites and attractions of scientific, historical and aesthetic importance that help to boost economic status of the metropolis, region and country at large. In spite of these, Cape Coast has an unemployment rate of $13 \%$ with unemployment affecting females more than males.

\subsection{Study Design}

The research was a cross-sectional study with questionnaires designed by the corresponding and the first authors to elicit some information from the study participants. The questionnaires were pretested on patients at Ewim District 
hospital, also at Cape Coast. After a few modifications, the questionnaires were administered based on the validity and reliability. There was actually no conflict of interest, with a well-structured closed-ended questionnaires administered to the HIV-positive antiretroviral therapy naive patients who were yet to be put on antiretroviral therapy. The demographic information obtained with the questionnaires was age, sex, occupation and level of education. Other variables obtained were, engagement in some risky behaviour such as smoking, drinking of alcohol, and having multiple sexual partners; and reactions towards health care given to them in the hospital laboratory.

\subsection{Ethical Consideration}

The study had approval from the Institutional Review Board, University of Cape Coast and the Cape Coast Teaching Hospital authorities. A verbal consent, as well as written informed consent was also sought from the participants of the study, after the procedures were clearly explained. Results and records were strictly kept confidential.

\subsection{Sample Collection}

Blood samples were collected from $150 \mathrm{HIV}$ infected patients who visited the Out Patients' Department of the Cape Coast Teaching Hospital. These patients were already diagnosed to be HIV positive and yet to be put on antiretroviral drugs. About $5 \mathrm{mls}$ of samples were collected with JMK Hypodermic syringes into P-Smart sterile EDTA tubes, and spun in the centrifuge to get the plasma for the various tests. All samples were assigned codes in order not to mix them or use for repeated data. The samples were tested for HIV with Rapid test kits and confirmed by ELISA as described by Baah et al. [16] [17]. Briefly, the plasma was reconstituted and mixed with the selenium colloid-antigen conjugate. A drop of the mixture formed was added to a spot on the strips to migrate through the solid phase to the antigen immobilized recombinant antigens and synthetic peptides at the patient window site. Antibodies to HIV-1 and/or HIV-2 present in the sample bound to the complex of antigen and antigen-selenium colloid conjugate forming a red line at the patient window site. However, the absence of antibodies to HIV-1 and/or HIV-2 allowed the antigen-selenium colloid moved past the patient window site, and no red line was formed. Also for the ELISA, a recombinant antigenic proteins and synthetic peptides from HIV-1, HIV-2, and HIV-1 group O were coated as discrete lines on a nylon strip with plastic backing. Plasma samples were incubated in a test trough together with the multiple antigen-coated test strips. HIV antibodies in the samples, bound to the individual HIV antigen lines on the strip. Afterwards, a goat anti-human IgG labelled with alkaline phosphatase was added to bind to any HIV antigen/antibody complex previously formed and incubation with the enzyme substrate (BCIP/NBT) produced a dark brown colour in proportion to the amount of HIV antibody present in the samples. Colour development was stopped with sulphuric acid. 
Samples containing no HIV-specific antibodies, the labelled antihuman antibody did not bind to antigen/antibody complex so that only a low standard background colour develops.

Syphilis test (VDRL) was carried out on HIV positive samples as described by Faustina et al. [18]. Briefly, the plasma was heat inactivated at $56^{\circ} \mathrm{C}$ for $30 \mathrm{~min}$ utes and diluted to 1 in 20. Samples were kept at room temperature for $30 \mathrm{~min}$ utes. About $25 \mu \mathrm{L}$ aliquots of plasma and $75 \mu \mathrm{L}$ antigen coated test cells, in a final dilution of 1 to 80 were transferred to $\mathrm{U}$ type microtitre plates examined for 4 hrs incubation at $25^{\circ} \mathrm{C}$. A final reading was made after overnight incubation. Results were recorded as follows: Negative-A smooth ring or button of cells. Positive-A diffuse carpet or a thin ring of cells with marked agglutination. Weak positive-A slightly enlarged ring of cells with peripheral agglutination.

\subsection{Statistical Analysis}

Data was entered into Microsoft Excel and statistical analysis was carried out using Statistical Package for Social Sciences (SPSS version 17, inc Chicago). Differences among participants groups (age, gender, occupation, marital status, risky sexual factors, and laboratory satisfaction) were analysed using Chi-square goodness-of-fit and fisher exact test. Collective co-efficient was calculated for variable of interest and the significance level set at 0.05 .

\section{Results}

Syphilis appears to be quite high in the Central Region of Ghana and out of 150 HIV positive participants tested, 15 (10\%) were positive for the disease. In relation to their demographic factors, Table 1 showed the age groups, sexes and marital status of the subjects involved in the study. It can be found that 10 (6.67\%) of the participants within the age group $20-29$ and $30-39$ years were infected with syphilis. These were followed by $40-49$ and $50-59$ age groups with 2 (1.33\%) each. There was no trace of Treponema palladium antibodies in the samples of participants in the age groups below 20 years $(0 \%)$. Therefore, the prevalence is significantly higher among $20-29$ and $30-39$ age groups in HIV positive antiretroviral therapy naive participants in Cape Coast $(\mathrm{p}=0.026)$ than the other age groups.

Among males and females it was noted that $6(4.00 \%)$ of the males had the traces of syphilis antibodies, similarly females who were infected were $9(6.00 \%)$ $(\mathrm{p}=0.828)$. Eighty $(53.33 \%)$ of males, and $70(46.67 \%)$ of females, were not infected with the bacteria.

Again, the prevalence of syphilis in married patients was $6(4.00 \%)$ and was similar in unmarried, $9(6 \%)$, participants, $(\mathrm{p}=0.828)$. Also $86(57.33 \%)$ of the married participants were not infected, just as $64(42.67 \%)$ of the unmarried participants were also negative to the infection.

A greater percentage of the participants have been through formal education to tertiary level. Majority of them had been to junior high school or senior high 
Table 1. The demographic data of HIV subjects with Syphilis infection.

\begin{tabular}{|c|c|c|c|}
\hline Syphilis status & Number (\%) & Positive (\%) & P-value \\
\hline \multicolumn{4}{|l|}{ Age group } \\
\hline$<20$ & $28(18.67)$ & $0(0.00)$ & 0.026 \\
\hline $20-29$ & $23(15.33)$ & $5(3.33)$ & \\
\hline $30-39$ & $56(37.33)$ & $5(3.33)$ & \\
\hline $40-49$ & $28(18.67)$ & $2(1.33)$ & \\
\hline $50-59$ & $14(9.33)$ & $2(1.33)$ & \\
\hline$\geq 60$ & $1(0.67)$ & $1(0.67)$ & \\
\hline Total & $150(100)$ & $15(10.00)$ & \\
\hline \multicolumn{4}{|l|}{ Sex } \\
\hline Male & $80(53.33)$ & $6(4.00)$ & 0.828 \\
\hline Female & $70(46.67)$ & $9(6.00)$ & \\
\hline Total & $150(100)$ & $15(10.00)$ & \\
\hline \multicolumn{4}{|l|}{ Marital status } \\
\hline Married & $86(57.33)$ & $6(4.00)$ & 0.828 \\
\hline Single & $64(42.67)$ & $9(6.00)$ & \\
\hline Total & $150(100)$ & $15(10.00)$ & \\
\hline \multicolumn{4}{|l|}{ Educational status } \\
\hline None & $21(14.00)$ & $0(0.00)$ & 0.241 \\
\hline Primary & $23(14.67)$ & $1(0.67)$ & \\
\hline JHS/Middle sch. & $43(28.67)$ & $5(3.33)$ & \\
\hline SHS & $61(40.67)$ & $5(3.33)$ & \\
\hline Tertiary & $2(1.34)$ & $1(0.67)$ & \\
\hline Total & $150(100)$ & $15(10.00)$ & \\
\hline
\end{tabular}

SHS: Senior High school, JHS: Junior High School.

school. However, there was no differences among educational status and syphilis infections $(\mathrm{p}=0.241)$.

The waiting times of the patients at the laboratory were determined to find out their satisfaction level (Table 2). It was noted that majority of the participants were satisfied with the activities of the laboratory personnel. However, there were no difference in syphilis positive participants in their response to satisfaction with laboratory personnel $(\mathrm{p}=0.337)$. Majority of the participants also were nicely received by the laboratory personnel with no difference in positive patients response as well $(\mathrm{p}=0.201)$. However, many of them were not satisfied with the waiting time at the laboratory but with no differences as well as in the syphilis positive patients $(\mathrm{p}=0.201)$.

From Table 3, the number of males $(p=0.0189)$ and females $(p=0.015)$ who 
Table 2. Assessment of Laboratory Services at the hospital.

\begin{tabular}{ccccc}
\hline \multicolumn{5}{c}{ Syphilis status } \\
\hline Satisfied with & Yes & $122(81.33)$ & $12(9.00)$ & 0.337 \\
Laboratory personnel & No & $28(18.67)$ & $3(2.00)$ & \\
Satisfaction with & Yes & $141(94.00)$ & $13(8.67)$ & 0.201 \\
Laboratory reception & No & $9(6.00)$ & $2(1.33)$ & \\
Satisfaction with & Yes & $48(32.00)$ & $13(8.67)$ & 0.201 \\
Waiting time at the & No & $102(68.00)$ & $2(1.33)$ & \\
Laboratory & & &
\end{tabular}

Table 3. The activities of the participants (predisposing factors) leading to syphilis infection.

\begin{tabular}{lccccc}
\hline \multicolumn{5}{c}{ Sex } \\
\hline & Male & p-value & Female & p-value \\
\hline Pes & $5(3.33)$ & 0.0189 & $4(2.67)$ & 0.015 \\
& No & $75(50.00)$ & & $66(44.00)$ & \\
$\begin{array}{c}\text { Participants Involved in } \\
\text { alcohol Intake }\end{array}$ & Yes & $26(17.33)$ & 0.603 & $11(7.33)$ & 0.199 \\
& No & $54(36.00)$ & & $59(39.33)$ & \\
Participants involved in & Yes & $4(2.67)$ & 0.128 & $11(7.33)$ & 0.002 \\
multiple sexual relation & No & $39(26.00)$ & & $58(38.67)$ & \\
& None & $37(24.67)$ & & $1(0.67)$ & \\
\hline
\end{tabular}

smoked were significantly lower than the corresponding sexes who did not smoked. However, there were no differences in alcohol intake among the males $(\mathrm{p}=0.603)$ and the females $(\mathrm{p}=0.199)$. Also there were no differences among the males $(p=0.128)$ in having multiple sexual relation but a significant fewer number of the females $(p=0.002)$ were having multiple sexual relations.

In Table 4, majority of the participants appreciated the need to carry out syphilis test among population to know their status. All those who were infected with the bacteria supported frequent testing of the infection among the population $(p=0.004)$. However, a significant number of them did not support the control of sexually transmitted infection during sexual relation $(p=0.022)$. Also majority of them did not know that smoking, alcohol intake and multiple sexual relation did related to acquisition of syphilis infection ( $p>0.05)$ (Table 5).

\section{Discussion}

From the study, the prevalence rate of syphilis in HIV infected patients at Cape Coast Metropolis was 10\%. This was higher compared to what was noted in Addis Ababa, with prevalence of 1.6\% infection [19] and 5.3\% syphilis prevalence among HIV positive patients in Iran [20]. The findings may imply syphilis infection could be among the most common opportunistic infections affecting the 
Table 4. Respondents' recommendation for syphilis test.

\begin{tabular}{lcccc}
\hline \multicolumn{5}{l}{ Syphilis status } \\
\hline & & Number (\%) & Positive (\%) & P-value \\
\hline \multirow{3}{*}{ Syphilis test Recommendation } & Yes & $148(98.67)$ & $15(10.00)$ & 0.004 \\
& No & $2(1.33)$ & $0(0.00)$ & \\
& Yes & $10(6.67)$ & $1(0.67)$ & 0.022 \\
Control of STIs In sexual relation & No & $136(92.00)$ & $14(9.33)$ & \\
& None & $4(2.67)$ & $0(0.00)$ & \\
\hline
\end{tabular}

Table 5. Association risk factor and syphilis infection.

\begin{tabular}{ccccc}
\hline Variables & Response & \multicolumn{3}{c}{ Syphilis } \\
\hline \multirow{2}{*}{ Smoking } & Yes & 9 & Positive (\%) & P-value \\
\hline \multirow{2}{*}{ Alcohol intake } & No & 141 & 1 & 0.074 \\
& Yes & 37 & 6 & 0.787 \\
Multiple sexual & No & 113 & 9 & \\
relation & Yes & 15 & 7 & 0.406 \\
& No & 97 & 7 & \\
\hline
\end{tabular}

HIV infected individuals in Cape Coast. Nevertheless, the prevalence of syphilis among the HIV patients in this study was lower than that of HIV positive antiretroviral naive adults attending APIN Clinic at Lagos University Teaching Hospital, being 20\% [21] and more so in co-infection of Syphilis and HIV in Port Harcourt, Nigeria, being 33\% [22]. It is therefore likely syphilis is a common opportunistic infection among HIV infected patients when they do not take precautionary measures in their sexual practices.

In addition, the infection was high among the youth ages between $20-39$ years. This is not surprising because youths of these age groups are sexually active and may be having more than one sexual partner. However, it was noted that a significant number of participants were not smoking, drinking or having multiple sexual relation. These suggest the above factors may not be exposing individuals to syphilis infection in the community.

The promiscuous behaviour of women in the society may be due to economic hardship especially among the young ladies who may be indulging in sexual activity for their livelihood. This assertion is supported by the fact that more females HIV infected patients were also infected with syphilis as compared with their male counterparts. Syphilis infection may be subclinical in females for a long time and that makes them spread the disease without knowing that they are infected. Unlike the females, majority of the males develop symptoms in early stages of infection and therefore seek medical attention [23]. More so, it has 
been proven that HIV positive patients have higher risk ( 3 - 5 folds or times) in contracting other sexually transmitted infections if one engages in multiple sex practices [24] [25]. Again, it has been noted from the study that more of the males are engaged in having multiple sexual partners compared to the females. However, more females were infected with both HIV and syphilis infections suggesting they may be actually having multiple sexual partners but did not disclose that in their questionnaires. Taking of alcohol and smoking are also a significant risk factors associated with STI infection. From the study, a few of both males and females were involved in smoking as well as taking alcohol supporting the fact that this did not contribute much into the acquisition of syphilis infection in the community.

Even though the males were of higher proportion as compared to the ladies they appeared to be protecting themselves against STI when engaging in sexual acts [20]. This may be due to the fact that more visible expression of symptoms of syphilis occurs in male genitalia as a compared to the female genitalia [23] and therefore the males are cautious in their sexual activity. Education is also important in creating awareness and control of syphilis/HIV infections, but it did not play a significant role in the rate of the infections.

Majority of infected participants recommended syphilis test within the community but they did not support the control of sexually transmitted infections among couples in relationship. This could be the fear of being known as infected participants and subsequent loss of sexual partners. Smoking, alcohol intake and multiple relations are known predisposing factors leading to infection of most sexually transmitted infections including syphilis [26]. This study has shown no association between these factors and syphilis. It is likely HIV might be the main factor that is contributing to the high prevalence of syphilis as recently noted in the Central Region of Ghana in which Cape Coast is the capital.

Majority of the participants including syphilis-infected patients were generally satisfied with the entire processes in the laboratory. However, smaller number of them was satisfied with the awaiting time at the hospital's laboratory, which is not encouraging at all. This can deter people from coming to the laboratory voluntarily or if the person feels he or she is healthy. Again those participants, who were negative for syphilis, may not be willing to attend the hospital for any laboratory check-up, if they contract the infection after their experienced with this laboratory session. Eventually, some members of the population may be harboring the bacteria for a longer time and will aid in spreading the bacteria among others, leading to shorter life expectancy or neurologic involvement [20]. It was noted that some of the participants complained of unfriendly reception and delay in services. Solution to these complaints is very necessary in promoting the good attitudes of members of the Cape Coast Metropolis in engaging in regular laboratory tests for STIs, thereby tracking the syphilis infection in its infectious stages [19]. Also further detailed studies may help understand the relationship between these infections among the youth of the population in the metropolis. 


\section{Conclusion}

In conclusion, the prevalence of syphilis in HIV positive antiretroviral therapy naive patients in Cape Coast is high and common among age groups of 20 - 39 years of the population. This is of great concerned since the youth are the back bone of the economy in the country and should be encouraged to lead sexually moral life.

\section{Acknowledgements}

We appreciate the contribution of Mr. E. Acheampong for his assistance in carrying out the laboratory work. Our sincere thanks go to staff of the Cape Coast teaching hospital laboratory for their help and guidance in bring the study to a successful end and also Mr. Koffie for assisting in statistical analysis of the data. We also thank the Dean of School of Biological Sciences, University of Cape Coast for supporting the study.

\section{Conflicts of Interest}

The authors declare no conflicts of interest regarding the publication of this paper.

\section{References}

[1] Zetola, N.M. and Klausner, J.D. (2007) Syphilis and HIV Infection: An Update. Clinical Infectious Diseases, 44, 1222-1228. https://doi.org/10.1086/513427

[2] Niama, R.F., Loukabou Bongolo, N.C., Bayonne Kombo, E.S., Yengo, R., Mayengue, P.I., Mandingha Kosso, E.B., et al. (2017) Syphilis and HIV Infections among Pregnant Women Attending Antenatal Clinics in Republic of Congo. The Pan African Medical Journal, 28, 8. https://doi.org/10.11604/pamj.2017.28.8.13097

[3] Newman, L., Rowley, J., Vander Hoorn, S., Wijesooriya, N.S., Unemo, M., Low, N., et al. (2015) Global Estimates of the Prevalence and Incidence of Four Curable Sexually Transmitted Infections in 2012 Based on Systematic Review and Global Reporting. PLoS One, 10, e0143304. https://doi.org/10.1371/journal.pone.0143304

[4] WHO (2016) Global Health Observatory (GHO) Data. 2016. Child Mortality and Causes of Death. WHO, Geneva.

[5] CDC (2016) Sexually Transmitted Diseases Surveillance. Centers for Disease Control and Prevention. https://www.cdc.gov/std/stats16/default.htm

[6] UNAIDS (2018) Global AIDS Monitoring 2018-Unaids. http://www.unaids.org/sites/default/files/media_asset/global-aids-monitoring_en.pdf

[7] UNAIDS (2018) Fact Sheet-Latest Global and Regional Statistics on the Status ... -Unaids. http://www.unaids.org/sites/default/files/media_asset/UNAIDS_FactSheet_en.pdf

[8] Mehta, S.D., Ghanem, K.G., Rompalo, A.M. and Erbelding, E.J. (2006) HIV Seroconversion among Public Sexually Transmitted Disease Clinic Patients: Analysis of Risks to Facilitate Early Identification. Journal of Acquired Immune Deficiency Syndromes, 42, 116-122. https://doi.org/10.1097/01.qai.0000200662.40215.34

[9] Baeten, J.M. and Overbaugh, J. (2003) Measuring the Infectiousness of Persons with HIV-1: Opportunities for Preventing Sexual HIV-1 Transmission. Current HIV Research, 1, 69-86. https://doi.org/10.2174/1570162033352110 
[10] Adjei, A.A., Atta, P.B., Krampa, F., Lartey, M., Rahman, M.A., Agyeman, S., et al. (2016) Human Immunodeficiency Virus, Hepatitis B Virus and Syphilis Infections among Long-Distance Truck Drivers in, a Port City in Ghana. African Journal of Infectious Diseases, 10, 10-16. https://doi.org/10.21010/ajid.v10i1.2

[11] Alomatu, H. (2016) HIV, HBV, HCV and Syphilis Infections among Blood Donors in Koforidua, Ghana. University of Ghana.

[12] Sarkodie, F., Ullum, H., Owusu-Dabo, E., Owusu-Ofori, S., Owusu-Ofori, A. and Hassall, O. (2016) A Novel Strategy for Screening Blood Donors for Syphilis at Komfo Anokye Teaching Hospital, Ghana. Transfusion Medicine, 26, 63-66. https://doi.org/10.1111/tme.12279

[13] Adjei, A.A., Armah, H.B., Gbagbo, F., Ampofo, W.K., Quaye, I.K., Hesse, I.F. and Mensah, G. (2006) Prevalence of Human Immunodeficiency Virus, Hepatitis B Virus, Hepatitis $\mathrm{C}$ Virus and Syphilis among Prison Inmates and Officers at Nsawam and Accra, Ghana. Journal of Medical Microbiology, 55, 593-597. https://doi.org/10.1099/jmm.0.46414-0

[14] NACP (2017) National AIDS Control Programme Releases 2016 HIV Sentinel Report. https://www.ghanabusinessnews.com

[15] GPS (2010) GPS Coordinates of Cape Coast, Ghana. Latitude: 5.1054 Longitude ... https://latitude.to/map/gh/ghana/cities/cape-coast

[16] Baah, A.T., Azumah, D.E., Ampiah, C., Boampong, J. and Nuvor, S.V. (2014) Incidence of Human Immunodeficiency Virus in Sickle Cell Patients in the Cape Coast Metropolis, Ghana. World Journal of AIDS, 4, 338-345. https://doi.org/10.4236/wja.2014.43040

[17] Tetteh-Attaah, R.L., Bonsu, E.A., Pappoe, F., Diabor, E., Dankwa, K. and Nuvor, S.V. (2016) Seroprevalence of Hyperglycaemia in HIV Positive Patients Visiting the Cape Coast Teaching Hospital in Ghana. Journal of Health, Medicine and Nursing, 33, 51-59.

[18] Faustina, N.-B.A., Dankwa, K., Ampiah, C., Boampong, J.N. and Nuvor, S.V. (2015) Seroprevalence of Syphilis Infection in Individuals at Cape Coast Metropolis, Ghana. BJMMR, 8, 157-164. https://doi.org/10.9734/BJMMR/2015/16267

[19] Eticha, B.T., Sisay, Z., Alemayehu, A. and Shimelis, T. (2013) Seroprevalence of Syphilis among HIV-Infected Individuals in Addis Ababa, Ethiopia: A Hospital-Based Cross-Sectional Study. BMJ Open, 3, e002566. https://doi.org/10.1136/bmjopen-2013-002566

[20] Badie, B.M., Yavari, Z., Esmaeeli, S., Paydary, K., Emamzadeh-Fard, S., Alinaghi, S.A.S. and Rasoulinejad, M. (2013) Prevalence Survey of Infection with Treponema pallidum among HIV-Positive Patients in Tehran. Asian Pacific Journal of Tropical Biomedicine, 3, 334-336. https://doi.org/10.1016/S2221-1691(13)60074-X

[21] Opara, M., Ogbebor, V., Fasasi, M., Akanmu, S., Bamiro, B., Ayolabi, C., et al. (2013) Incidences of Hepatitis B and Syphilis Co-Infection with HIV in Antiretroviral Treatment-Näve Adult Patients Attending APIN Clinic at a University Teaching hospital in Lagos, Nigeria. Journal of AIDS and Clinical Research, 4, No. 1.

[22] Tessema, B., Yismaw, G., Kassu, A., Amsalu, A., Mulu, A., Emmrich, F. and Sack, U. (2010) Seroprevalence of HIV, HBV, HCV and Syphilis Infections among Blood Donors at Gondar University Teaching Hospital, Northwest Ethiopia: Declining Trends over a Period of Five Years. BMC Infectious Diseases, 10, 111. https://doi.org/10.1186/1471-2334-10-111

[23] Sellati, T.J., Wilkinson, D.A., Sheffield, J.S., Koup, R.A., Radolf, J.D. and Norgard, M.V. (2000) Virulent Treponema pallidum, Lipoprotein, and Synthetic Lipopep- 
tides Induce CCR5 on Human Monocytes and Enhance Their Susceptibility to Infection by Human Immunodeficiency Virus Type 1. The Journal of Infectious Diseases, 181, 283-293. https://doi.org/10.1086/315209

[24] Simaleko, M.M., Longo, J.D.D., Police, S.M.C., Grésenguet, G., Bouassa, R.S.M., Piette, D., et al. (2018) Persistent High-Risk Behavior and Escalating HIV, Syphilis and Hepatitis B Incidences among Men Who Have Sex with Men Living in Bangui, Central African Republic. Pan African Medical Journal, 29, 132.

https://doi.org/10.11604/pamj.2018.29.132.12794

[25] Johnston, L., Vaillant, T., Dolores, Y. and Vales, H. (2013) HIV, Hepatitis B/C and Syphilis Prevalence and Risk Behaviors among Gay, Transsexuals and Men Who Have Sex with Men, Dominican Republic. International journal of STD \& AIDS, 24, 313-321. https://doi.org/10.1177/0956462412472460

[26] Aydin, Ö.A., Karaosmanoglu, H.K., Sayan, M., Ince, E.R. and Nazlican, Ö. (2015) Seroprevalence and Risk Factors of Syphilis among HIV/AIDS Patients in Istanbul, Turkey. Central European Journal of Public Health, 23, 65.

https://doi.org/10.21101/cejph.a4001 


\section{Appendix}

The questionnaire used in this study

NB: Please, tick the correct answers, and fill in the spaces where necessary.

SECTION A: SOCIO-DEMOGRAPHIC PROFILE

AGE: a. Less than 20 years [ ] b. $20-29$ years [ ] c. $30-39$ years [ ] d. 40

- 49 years [ ] e. 50 - 59 years [ ] f. 60 and above [ ]

SEX: a. Male [ ] b. Female [ ]

EDUCATIONAL STATUS: a. None [ ] b. Primary [ ] c. JHS/Middle school [ ]d.SHS [ ] e. Tertiary [ ]

MARITAL STATUS: a. Single [ ] b. Married [ ]

OCCUPATION: a. Unemployed [ ] b. Trading [ ] c. Student [ ] d. Civil servant/public servant [ ] e. Others (specify)

SECTION B: SELECTED RISKY SOCIAL BEHAVIOURS

Do you take in alcohol? A. Yes [ ] B. No [ ]

If "YES", how often? A. Occasionally [ ] B. Daily/Habitually [ ] C. 2 - 5 days within the week [ ]

Do you smoke? A. Yes [ ] B. No [ ]

If "YES", how often? A. Occasionally [ ] B. Daily/Habitually [ ] C. 2 - 5 days within the week [ ]

Do you have multiple sex partners? A. Yes [ ] B. No [ ]

If "YES", how often do you control each other's self against sexual transmitted infections? A. Occasionally [ ] B. All the time [ ]

SECTION C: KNOWLEDGE ABOUT SYPHILIS

Have you heard about syphilis? A. Yes [ ] B. No [ ]

Do you have any idea about it? A. Yes [ ] B. No [ ]

Have you ever been tested for it? A. Yes [ ] B. No [ ]

If "YES" to both 12 and 13, but "NO" to 14 , why?

\section{SECTION D: SUBJECTS SATISFACTION TO HEALTH CARE}

\section{PRACTICES}

Is today your first visit to this hospital? A. Yes [ ] B. No [ ]

Are you satisfied with the reception by the laboratory personnel? A. Yes [ ]

B. No [ ]

If "NO", why?

Are you satisfied with the waiting time before taking your test? A. Yes [ ] B. No [ ]

Are satisfied with the laboratory's reception? A. Yes [ ] B. No [ ]

If "NO", why?

How was the attitude of the laboratory personnel towards you? A. Good [ ]

B. Poor [ ] 
Did you enjoy privacy during the take of test? A. Yes [ ] B. No [ ]

If "NO", why?

What was the attitude of the laboratory technologist towards you? A. Good [ ] B. Poor [ ]

Would you recommend syphilis test to be made to others? A. Yes [ ] B. No [ ]

Are there any things you want the hospital to put in place to make patients satisfied? A. Yes [ ] B. No [ ]

If "Yes", what are they?

Overall, how satisfied were you with your visit today? A. Very satisfied [ ] B. Satisfied [ ] C. Dissatisfied [ ]

NB: Thanks a lot for your cooperation and the promise of confidentiality will still hold in the highest esteem. We hope to attend to all complaints given. 